\title{
Programm- und Softwareentwicklung für die Grundwassermodellierung
}

\author{
Olaf Kolditz • Thomas Kalbacher • Wenqing Wang
}

Online veröffentlicht: 21.7 .2010

(C) Springer-Verlag 2010

Es ist einsam geworden ... Als der Erstautor seine Doktorarbeit schrieb (es ist schon eine geraume Zeit her), gab es hierzulande mindestens 10 Forschungseinrichtungen, die sich mit der Methoden- und Programmentwicklung für die Grundwassermodellierung intensiv beschäftigten. Dass es nun weniger geworden sind, hat nicht so sehr mit der deutschen Wiedervereinigung zu tun, sondern ist ein logischer Globalisierungsprozess. Man will immer komplexere Fragestellungen angehen, damit steigt der Aufwand erheblich und so ist die Landschaft der wissenschaftlichen und kommerziellen Software mittlerweile eben fokussierter und recht überschaubar geworden.

Es ist aber auch anders geworden ... Neben kommerzieller Programmentwicklung und den vielfach unbeachteten, projektspezifischen Einzellösungen gibt es eine dritte Richtung unter Verwendung von soliden kommerziellen, skriptbasierten Tools zur numerischen Lösung von Problemen. Mit relativ wenig Programmieraufwand kommt man rasch zu Testbausteinen, die eine Antwort auf eine spezielle wissenschaftliche Fragestellung liefern und kann somit Kernpunkte identifizieren, die es lohnt weiter zu verfolgen, oder man merkt schnell, dass man auf dem Holzweg ist.

Als vierte Möglichkeit gibt es noch die wissenschaftliche Softwareentwicklung. Sie ermöglicht die Beantwortung komplexerer Fragestellungen (z. B. Prozesskopplun-

Prof. Dr. O. Kolditz · Dr. T. Kalbacher $(\varangle) \cdot$ Dr. W. Wang

Department Umweltinformatik, Helmholtz-Zentrum für

Umweltforschung GmbH - UFZ, Permoserstraße 15,

04318 Leipzig, Deutschland

E-Mail: thomas.kalbacher@ufz.de

Prof. Dr. O. Kolditz

E-Mail: olaf.kolditz@ufz.de

Dr. W. Wang

E-Mail: wenqing.wang@ufz.de gen, nichtlineare Systeme, Unsicherheitsanalysen und realitätsnahe Modellgeometrien). Der programmtechnische Aufwand für die Entwickler steigt dabei exponentiell, da große Teile der Software - insbesondere die Numerik - komplett selber geschrieben werden. In den Codes stecken oft viele Dekaden Entwicklung und das enthaltene Know-How ist daher beträchtlich. „Überlebt“ haben Codes, welche sich stets neue Softwarearchitekturen und Sprachen zu Nutze gemacht und Software-Engineering integriert haben. Dabei wurden Codes zum Teil alle 5 Jahre völlig neu geschrieben und dies zeigt, dass die Handhabung solcher ,nicht zur Ruhe kommender" Software nichts für schwache Nerven ist. Der Vorteil liegt in der Flexibilität, der einfachen Wiederverwendung von zahlreichen Schnittstellen zum Prä- und Postprozessing, den Möglichkeiten zum Parallelrechnen oder zur modernen Visualisierung und vor allem in der Zusammenführung gleichgesinnter Gesprächspartner und Interessensgruppen.

Die wissenschaftliche Software spielt folglich auch eine zentrale Rolle bei der Kommunikation in der Forschung und dies ist insbesondere dann der Fall, wenn die Codes als Open-Source-Projekte zur Verfügung gestellt werden. Dies ermöglicht die gegenseitige Bewertung von Erfolgen (und auch Misserfolgen) und viele der oben genannten kreativen Einzellösungen und Testbausteine verdienen es, in größere Softwareprojekte aufgenommen zu werden. Die wissenschaftliche Software ist damit ein öffentlich zugänglicher Ideenpool. Das Potenzial für neue Produkte ist also riesig und stellt eine attraktive Herausforderung für die hydrogeologische Szene dar. 